\title{
Effect of Non-Steroidal Anti-Inflammatory Drugs on Methotrexate Dosage, Corticosteroid Dosage, Rheumatoid Arthritis Activity and Laboratory Data in Patients with Rheumatoid Arthritis
}

\author{
Norio Masumoto*1, Yoshihisa Takao, Kazutaka Harada ${ }^{2}$ and Seigo Iwakawa ${ }^{3}$ \\ Departments of Pharmacy ${ }^{1}$ and Orthopedics ${ }^{2}$, Kawanishi City Hospital, \\ Department of Pharmaceutics, Kobe Pharmaceutical University ${ }^{3}$ \\ [ Received January 31, 2012 ] \\ Accepted May 3, 2012
}

The effect of non-steroidal anti-inflammatory drugs (NSAIDs) on methotrexate (MTX) dosage, corticosteroid dosage, rheumatoid arthritis (RA) activity and laboratory data in MTX-treated patients with RA was investigated retrospectively. The MTX dosage was significantly increased in $67 \%$ of the control patients, while in only $33 \%$ of the NSAID-treated patients, MTX dosage was increased $(p<0.05)$. A significant reduction of corticosteroid dosage $(p<0.05)$ and RA activity $(p<0.01)$ in the control patients was observed. Changes in several laboratory data of RA patients during the observation period were similar between the two patient groups.

Key words — methotrexate, corticosteroid, non-steroidal anti-inflammatory drugs, dosage, rheumatoid arthritis

\section{Introduction}

Patients with rheumatoid arthritis (RA) are usually treated with a combination of non-steroidal anti-inflammatory drugs (NSAIDs), corticosteroids, and disease-modifying anti-rheumatic drugs (DMARDs). The early use of DMARDs such as methotrexate (MTX) after diagnosing RA is recommended according to the recent guideline for the diagnosis and treatment of RA in Japan. ${ }^{1,2)}$ The immunosuppressive and anti-inflammatory effects of MTX show pathological improvement of the synovial membranes in RA patients. ${ }^{3)}$ Serious adverse effects by drug interactions with MTX include interstitial pneumonia, bone-marrow suppression, infectious disease, and hepatic disorders were reported. ${ }^{4,5)}$ In terms of MTX dosage, more than $6 \mathrm{mg} / \mathrm{week}$ accounts for $61.6 \%$ among the fatal adverse cases reported in Japan. ${ }^{5}$ Several NSAIDs suppress the uptake of MTX using the organic anion transporter (OAT) and multidrug resistance-associated protein by the kidney. ${ }^{6}$ Elevated blood concentration of MTX by the administration of NSAIDs has been reported with high-dose (more than $1000 \mathrm{mg}$ ) MTX for the treatment of acute leukemia and malignant lymphoma. ${ }^{7}$ MTX at a low dosage co-treated with NSAIDs for RA patients also caused cytopenia. ${ }^{8}$ Meanwhile, it has also been reported that the interaction of MTX and NSAIDs would be insignificant if NSAIDs were used together at a low dosage of MTX. ${ }^{9}$ Few reports have discussed the effect of anti-inflammatory drugs on MTX dosage changes. In this study, we retrospectively investigated the effect of NSAIDs on MTX dosage changes in RA patients. Because corticosteroids

* 5-21-1, Higashiuneno, Kawanishi-shi, Hyogo, 666-0195 Japan 
are used together in most RA patients during MTX treatment, the dosage change in corticosteroids was also investigated.

Disease Activity Score 28-c-reactive protein $(\mathrm{DAS} 28-\mathrm{CRP})^{10)}$ is one of the most popular indicator of RA activity, therefore we chose DAS 28CRP as the index of RA activity in this study. As the index of adverse effects, the laboratory data of these patients were also monitored during combination therapy.

\section{Materials and Methods}

\section{Subjects and study period}

Patients, prescribed MTX at Kawanishi City Hospital from April 2005 to April 2009, were extracted from the ordering system, and were followed from July 2000 to April 2009. The MTX dosage of patients who were treated with NSAIDs daily (NSAID group) was compared with patients who were not treated with NSAIDs (control group) during the period of MTX administration in RA patients. Sixty-nine patients, prescribed MTX with NSAIDs (NSAID group; $\mathrm{n}=18$ ) or without NSAIDs (control group; $\mathrm{n}=51$ ), were extracted. In the NSAID group, loxoprofen sodium $(n=9)$, diclofenac sodium $(n=4)$, etodolac $(n$ $=2)$, aspirin $(n=1)$, meloxicam $(n=1)$, or lornoxicam $(n=1)$ were used. Predonisolone $(n=$ $63)$ or betamethasone $(n=2)$ were used as corticosteroids. Excluded cases were patients admitted from other hospitals, patients whose MTX administration period was less than three months, and patients who interrupted the administration of NSAIDs during the investigation period. Our present study was approved by the ethics committee at our hospital according to the ethics guideline concerning epidemiological studies.

\section{Investigated items}

We investigated the following items during the administration of MTX to RA patients.

1) Patient characteristics (sex, age, MTX treatment period)

2) Dosage of MTX

3) Dosage of corticosteroid

4) DAS 28-CRP

5) Laboratory data (aspartate aminotransferase (AST), alanine aminotransferase (ALT), blood urea nitrogen (BUN), estimated glomerular filtration rate (eGFR), white blood cells (WBC), hemoglobin $(\mathrm{Hb})$, and platelets (PLT))

\section{Statistical analysis}

The RA treatment period was expressed as the median, and other patient characteristic parameters were expressed as the mean \pm standard deviation. The MTX dosage, corticosteroid dosage, DAS 28-CRP and the laboratory data were expressed as the mean \pm standard deviation. The ratio of patients whom MTX dosage increased between the start of administration and the end of observation were compared using the chi-square test between two groups. The others were compared using Student's $t$-test. The level of significance was $p<0.05$.

\section{Results}

\section{Patient characteristics}

The characteristics of RA patients investigated are shown in Table 1. The RA treatment period (median) was 7.8 years in the NSAID group and 3.0 years in the control group. The NSAID group had a longer MTX treatment period than the control group. The dosage of MTX was $4.0 \pm 1.2 \mathrm{mg} /$ week in the NSAID group and $3.8 \pm 0.7 \mathrm{mg} /$ week in the control group. The dosage of corticosteroid 
Table 1 Patient characteristics

\begin{tabular}{lcc}
\hline \hline & $\begin{array}{c}\text { Control Group } \\
(\mathrm{n}=51)\end{array}$ & $\begin{array}{c}\text { NSAID Group } \\
(\mathrm{n}=18)\end{array}$ \\
\hline Sex (Male/Famale) & $12 / 39$ & $4 / 14$ \\
Age (years) & $58.1 \pm 11.0$ & $63.4 \pm 12.1$ \\
RA treatment period (median) & 3.0 & 7.8 \\
(years; range) & $(0-23.0)$ & $(0-21.0)$ \\
Observation period of & $2.5 \pm 1.8$ & $2.0 \pm 1.1$ \\
MTX administration (years) & $3.8 \pm 0.7$ & $4.0 \pm 1.2$ \\
MTX dosage (mg/week) & $4.2 \pm 1.7$ & $4.6 \pm 3.3$ \\
Corticosteroid dosage ${ }^{\mathrm{a}, \mathrm{b}}$ (mg/day) & $3.0 \pm 0.8$ & $3.2 \pm 1.0$ \\
DAS 28-CRP c & 4 & 2 \\
RA Stage ${ }^{\mathrm{d}}$ I & 16 & 1 \\
\multicolumn{1}{|l}{ II } & 19 & 7 \\
\multicolumn{1}{|l}{ IV } & 12 & 8 \\
\hline
\end{tabular}

Mean \pm S.D.

NSAID : non-steroidal anti-inflammatory drug, RA : rheumatoid arthritis, MTX : methotrexate, DAS 28-CRP : disease activity score 28 -c-reactive protein.

a Number of patients who took corticosteroids was 47 in the control group and 18 in the NSAID group.

b Prednisolone equivalent.

c Number of patients whose DAS 28-CRP investigated was 49 in the control group and 15 in the NSAID group.

d Steinblocker's stage.

was $4.6 \pm 3.3 \mathrm{mg} /$ day in the NSAID group $(\mathrm{n}=$ 18 ) and $4.2 \pm 1.7 \mathrm{mg} /$ day in the control group (n $=47$ ). DAS 28 -CRP was $3.2 \pm 1.0$ in the NSAID group $(\mathrm{n}=15)$ and $3.0 \pm 0.8$ in the control group $(n=49)$. Between the two groups, there were insignificant differences in the period of MTX administration, in the dosage of MTX and corticosteroid or in DAS 28-CRP. The patients in the NSAID group classified by Steinblocker's RA stages were mainly in stage III and stage IV, while the patients in the control group were distributed in stage II, stage III and stage IV.

\section{Effect of NSAIDs on MTX dosage, cor- ticosteroid dosage and DAS 28-CRP in}

\section{RA patients}

The dosage of MTX was markedly increased from the start of administration $(3.8 \pm 0.7 \mathrm{mg}$ / week) to the end of observation $(5.7 \pm 1.7 \mathrm{mg} /$ week) in the control group $(p<0.01)$. In contrast, the dosage of MTX in the NSAID group was slightly increased from the start of administration $(4.0 \pm 1.2 \mathrm{mg} /$ week) to the end of observation (4.8 $\pm 1.7 \mathrm{mg} /$ week $)(p<0.05)$ (Table 2). In the control group, a marked increase in MTX dosage was observed in the RA stage II, III and IV. The ratio of patients with an increased MTX dosage was $67 \%(34 / 51)$ in the control group, as compared with $33 \%(6 / 18)$ in the NSAID group $(p<0.05)$. The proportion of patients for whom an increase of the MTX dosage was necessary in the NSAID group was therefore one half of the control group.

Corticosteroid dosage was insignificantly changed at the end of observation in the NSAID group, while there was a significant dosage reduction of corticosteroids in the control group $(p<$ 0.05) (Table 2). In the control group, a marked decrease in corticosteroids dosage was observed in the RA stage II. In the stage IV of the NSAID group, corticosteroid dosage was maintained at 6 $7 \mathrm{mg} /$ day.

DAS 28-CRP was insignificantly changed at 
Table 2 Effect of NSAIDs on MTX dosage (mg/week), corticosteroid dosage (mg/day) and DAS 28-CRP in RA patients

\begin{tabular}{|c|c|c|c|c|c|c|c|c|}
\hline & \multirow[b]{2}{*}{$\mathrm{n}$} & \multirow[b]{2}{*}{$\begin{array}{c}\text { MTX } \\
\text { treatment }\end{array}$} & \multicolumn{2}{|c|}{$\begin{array}{l}\text { MTX dosage }^{a} \\
(\mathrm{mg} / \text { week })\end{array}$} & \multicolumn{2}{|c|}{$\begin{array}{c}\text { Corticosteroid dosage } \mathrm{e}^{\mathrm{b}, \mathrm{c}} \\
\text { (mg/day) }\end{array}$} & \multicolumn{2}{|c|}{ DAS $28-C R P d$} \\
\hline & & & Start & End & Start & End & Start & End \\
\hline Control Group & 51 & & $3.8 \pm 0.7$ & $5.7 \pm 1.7 * *$ & $4.2 \pm 1.7$ & $3.5 \pm 2.2 *$ & $3.0 \pm 0.8$ & $2.6 \pm 0.9 * *$ \\
\hline RA Stage I & 4 & & $4.0 \pm 0.0$ & $5.5 \pm 1.0$ & $5.0 \pm 0.0$ & $5.0 \pm 3.7$ & $3.8 \pm 0.3$ & $3.8 \pm 1.2$ \\
\hline II & 16 & & $4.1 \pm 0.5$ & $5.9 \pm 2.0 * *$ & $4.1 \pm 2.0$ & $2.5 \pm 1.7 * *$ & $2.9 \pm 1.0$ & $2.4 \pm 0.5$ \\
\hline III & 19 & & $3.7 \pm 0.8$ & $5.2 \pm 1.5 * *$ & $4.1 \pm 1.1$ & $3.6 \pm 1.6$ & $2.8 \pm 0.8$ & $2.4 \pm 0.9 *$ \\
\hline IV & 12 & & $3.7 \pm 0.8$ & $6.2 \pm 1.6 * *$ & $4.2 \pm 2.6$ & $4.1 \pm 2.6$ & $3.1 \pm 0.8$ & $2.8 \pm 0.9$ \\
\hline NSAID Group & 18 & & $4.0 \pm 1.2$ & $4.8 \pm 1.7 *$ & $4.6 \pm 3.3$ & $5.1 \pm 3.0$ & $3.2 \pm 1.0$ & $2.9 \pm 1.0$ \\
\hline RA Stage I & 2 & & $5.0 \pm 1.4$ & $7.0 \pm 1.4$ & $4.0 \pm 1.4$ & $5.5 \pm 0.7$ & $2.3 \pm 1.3$ & $4.3 \pm 1.3$ \\
\hline II & 1 & & 4.0 & 6.0 & 2.0 & 0.0 & 2.2 & 2.5 \\
\hline III & 7 & & $4.3 \pm 1.4$ & $5.1 \pm 1.6$ & $3.1 \pm 2.8$ & $3.4 \pm 2.1$ & $3.3 \pm 1.0$ & $2.4 \pm 0.7$ \\
\hline IV & 8 & & $3.5 \pm 0.9$ & $3.8 \pm 1.3$ & $6.3 \pm 3.6$ & $7.1 \pm 2.6$ & $3.5 \pm 0.9$ & $2.8 \pm 1.0$ \\
\hline
\end{tabular}

\section{Mean \pm S.D.}

NSAID : non-steroidal anti-inflammatory drug, RA : rheumatoid arthritis, MTX : methotrexate, DAS 28-CRP : disease activity score 28-c-reactive protein.

a Number of patients who were prescribed MTX was 51 in the control group and 18 in the NSAID group.

b Number of patients who were prescribed corticosteroids was 47 in the control group and 18 in the NSAID group

c Prednisolone equivalent.

d Number of patients whose DAS 28-CRP investigated was 49 in the control group and 15 in the NSAID group.

Significantly different from Start $(* p<0.05)(* * p<0.01)$.

the end of observation in the NSAID group, while there was a significant reduction of DAS 28-CRP in the control group $(p<0.01)$ (Table 2). Especially, a marked decrease in DAS 28-CRP was observed in the RA stage III of the control group.

\section{Changes in laboratory data in RA pa- tients}

There were insignificant differences in the laboratory data, such as liver function (AST, ALT), renal function (BUN, eGFR), and bone marrow function (WBC, Hb, PLT) between the two groups for 30 months after MTX initiation (Table 3).

\section{Discussion}

We investigated the effect of NSAIDs on the MTX dosage, corticosteroid dosage, RA activity and laboratory data in RA patients. The RA treatment period (median) was 3.0 years in the control group, while 7.8 years in the NSAID group, which suggested a longer treatment period in the
NSAID group than in the control group. RA patients had been treated according to a pyramid method in which NSAIDs were chosen as the first step medicines of RA treatment. In the next step, corticosteroids or DMARDs were chosen in the pyramid method. However, after the introduction of RA management guideline by American College of Rheumatology in 2002, ${ }^{11)}$ DMARDs are chosen for early stage RA patients at Kawanishi City Hospital. The therapeutic efficacy of RA can be expected by the combination of MTX with corticosteroids, because using corticosteroids during MTX therapy accounted for 94\% (65/69) of the entire MTX-administrated patients. As for biological medicine, only etanercept is used in our hospital. No case switched from MTX treatment to etanercept treatment in the 69 cases studied. RA stages in the NSAID group were distributed in stage III and stage IV, which also suggest RA treatment period was longer in the NSAID group than in the control group.

The starting dosage of MTX was about $4 \mathrm{mg} /$ 
Table 3 Effect of NSAIDs on laboratory data in RA patients

\begin{tabular}{|c|c|c|c|}
\hline & \multicolumn{2}{|c|}{ MTX treatment } & \multirow{2}{*}{$p$-value } \\
\hline & Start & End * & \\
\hline \multicolumn{4}{|c|}{ Aspartate aminotransferase (AST)(IU/L) } \\
\hline Control Group & $17.6 \pm 5.4$ & $20.6 \pm 7.5$ & 0.082 \\
\hline NSAID Group & $17.4 \pm 4.0$ & $20.2 \pm 8.2$ & 0.496 \\
\hline \multicolumn{4}{|c|}{ Alanine aminotransferase (ALT))(IU/L) } \\
\hline Control Group & $14.1 \pm 6.6$ & $17.1 \pm 7.6$ & 0.097 \\
\hline NSAID Group & $12.7 \pm 3.8$ & $17.8 \pm 7.0$ & 0.183 \\
\hline \multicolumn{4}{|c|}{ Blood urea nitrogen $(\mathrm{BUN})(\mathrm{mg} / \mathrm{dL})$} \\
\hline Control Group & $14.6 \pm 3.5$ & $14.5 \pm 3.2$ & 0.913 \\
\hline NSAID Group & $17.3 \pm 4.8$ & $17.8 \pm 5.9$ & 0.876 \\
\hline \multicolumn{4}{|c|}{ Estimated glomerular filtration rate $(\mathrm{eGFR})\left(\mathrm{mL} / \mathrm{min} / 1.73 \mathrm{~m}^{2}\right)$} \\
\hline Control Group & $86.3 \pm 18.9$ & $79.5 \pm 15.7$ & 0.107 \\
\hline NSAID Group & $77.6 \pm 22.0$ & $67.8 \pm 19.8$ & 0.374 \\
\hline \multicolumn{4}{|c|}{ White blood cells $(\mathrm{WBC})\left(\times 10^{2} / \mu \mathrm{L}\right)$} \\
\hline Control Group & $75.6 \pm 19.1$ & $71.8 \pm 22.6$ & 0.474 \\
\hline NSAID Group & $91.4 \pm 32.1$ & $74.1 \pm 21.3$ & 0.186 \\
\hline \multicolumn{4}{|c|}{ Hemoglobin (Hb) (g/dL) } \\
\hline Control Group & $12.0 \pm 1.5$ & $12.5 \pm 1.6$ & 0.159 \\
\hline NSAID Group & $11.6 \pm 1.8$ & $11.7 \pm 1.8$ & 0.871 \\
\hline \multicolumn{4}{|c|}{ Platelets (PLT) $\left(\times 10^{4} / \mu \mathrm{L}\right)$} \\
\hline Control Group & $31.1 \pm 10.0$ & $28.2 \pm 7.7$ & 0.170 \\
\hline NSAID Group & $30.5 \pm 13.1$ & $28.7 \pm 9.5$ & 0.737 \\
\hline
\end{tabular}

Mean \pm S.D.

NSAID : non-steroidal anti-inflammatory drug.

* Observation period: 3 - 30 months.

week in both the NSAID and control groups. The relatively lower starting dosage of MTX is aimed to maintain organ function, such as bone marrow function in elderly patients. The dosage of MTX showed a marked increase during the investigation period in the control group $(p<0.01)$. The increase in MTX dosage can be avoided by using NSAIDs together with MTX. In 47\% (16 of 34 cases) of the MTX-increased patients in the present study, MTX increased during a period of less than 3 months. Meanwhile, in the NSAID group, NSAIDs had already been administrated for a long time before MTX was prescribed; therefore, it is conceivable that the anti-inflammatory action of NSAIDs might enable the control of RA activity without increasing MTX dosage.

A significant reduction of corticosteroid dosage was shown from $4.2 \mathrm{mg} /$ day at the start to $3.5 \mathrm{mg} /$ day at the end of observation in the control group $(p<0.05)$. A previous study by Kawasaki et al. re- ported that the steroid dosage of 25\% RA patients administered MTX was decreased. ${ }^{12)}$ There are other reports that corticosteroid dosage was reduced during MTX treatment in RA patients. ${ }^{13-15)}$ In the treatment guideline of RA, the steroid dosage is gradually reduced by $1 \mathrm{mg}$ every 1 to 2 months, when the control of RA activity is stable. ${ }^{1)}$ These results indicate a decrease of the corticosteroid dosage during MTX treatment.

Less than 2.6 of DAS 28-CRP indicates clinical remission of RA activity. A significant reduction of DAS 28-CRP was shown from 3.0 at the start to 2.6 at the end of observation in the control group $(p<0.01)$, which indicate that MTX treatment led RA activity to clinical remission in about half of RA patients of the control group.

Serious adverse reactions caused by MTX treatment were reported interstitial pneumonia, bone-marrow suppression, infectious disease, and hepatic disorders. ${ }^{5)}$ In the present study, MTX did 
not cause such serious symptoms from the start to the end of the observation period between the two groups. The laboratory data of these patients were insignificant differences during the 30 months observation period.

We evaluated the correlation of DAS 28-CRP, MTX and corticosteroid dosage classified into two groups, respectively (Table 4). Subgroup analysis was performed according to their DAS 28-CRP which measured every 3 months. DAS 28-CRP of less than 2.6 was $40-50 \%$ of the investigated data in the two groups, indicating that control of RA activity was maintained in about the half of the patients. The percentage of observation points in RA patients administered more than $6 \mathrm{mg} /$ week MTX dosage was 38.9\% in the control group and $6.3 \%$ in the NSAID group; therefore, the MTX dosage can be maintained at a lower level by the co-treatment of NSAIDs with MTX than by MTX treatment without NSAIDs.

As a preliminary analysis, we investigated the effect of MTX dosage and corticosteroid dosage on DAS 28-CRP with multiple regression analysis, the effect of corticosteroid dosage on DAS 28-CRP was significant in the control group $(\mathrm{n}=$ 30, regression coefficient: $0.23,95 \%$ range: 0.09 $0.37, p<0.01)$. However, we could not obtain the results which suggest the relationship between
MTX dosage and DAS 28-CRP. This study is a retrospective study from the data of various RA stage patients. Therefore a prospective study which is controlled by the similar background RA patients will be necessary to clarify the relationship among MTX dosage, corticosteroid dosage and DAS 28-CRP.

The increase of MTX blood concentration resulted from inhibiting renal uptake of MTX (via OAT) by NSAIDs has been reported. ${ }^{6,7)}$ Nozaki et al. examined the $K_{\mathrm{i}}$ values of NSAIDs for OAT1 and OAT3 to evaluate the inhibitory effect of NSAIDs on the uptake of MTX by human kidney slices. ${ }^{6}$ In this study, loxoprofen sodium $(n=9)$ was the most prescribed NSAIDs. There were insignificant difference in MTX dosage, corticosteroid dosage, DAS 28-CRP and laboratory data between loxoprofen sodium and other NSAIDs. The administration of loxoprofen sodium did not change the urine volume and GFR in elderly patients. ${ }^{16)}$ Moreover, it has been also reported that the concomitant administration of NSAIDs with MTX to inflammatory arthritis patients showed insignificant difference in the urinary excretion of $\alpha$-glutathione S-transferase, a marker of renal tubular injury, or albumin, when compared with MTX monotherapy. ${ }^{17}$

The decline of renal blood flow by NSAIDs

Table 4 Effect of NSAIDs on DAS 28-CRP, MTX dosage and corticosteroid dosage in RA patients

\begin{tabular}{|c|c|c|c|c|c|c|c|c|c|c|}
\hline \multirow{3}{*}{$\begin{array}{l}\text { MTX } \\
\text { Corticosteroid }^{\mathrm{a}}\end{array}$} & \multicolumn{5}{|c|}{ Control group } & \multicolumn{5}{|c|}{ NSAID group } \\
\hline & \multicolumn{2}{|c|}{$<6 \mathrm{mg} /$ week } & \multicolumn{2}{|c|}{$\geq 6 \mathrm{mg} /$ week } & \multirow{2}{*}{ Summation } & \multicolumn{2}{|c|}{$<6 \mathrm{mg} /$ week } & \multicolumn{2}{|c|}{$\geq 6 \mathrm{mg} /$ week } & \multirow{2}{*}{ - Summation } \\
\hline & $<5 \mathrm{mg} / \mathrm{d}$ & $\geq 5 \mathrm{mg} / \mathrm{d}$ & $<5 \mathrm{mg} / \mathrm{d}$ & $\geq 5 \mathrm{mg} / \mathrm{d}$ & & $<5 \mathrm{mg} / \mathrm{d}$ & $\geq 5 \mathrm{mg} / \mathrm{d}$ & $<5 \mathrm{mg} / \mathrm{d}$ & $\geq 5 \mathrm{mg} / \mathrm{d}$ & \\
\hline \multicolumn{11}{|l|}{ DAS 28-CRP } \\
\hline$<2.6$ & $18.2 \%$ & $10.3 \%$ & $6.5 \%$ & $6.5 \%$ & $41.5 \%$ & $6.2 \%$ & $35.4 \%$ & $4.2 \%$ & $2.1 \%$ & $47.9 \%$ \\
\hline$\geq 2.6$ & $16.9 \%$ & $15.7 \%$ & $14.9 \%$ & $11.0 \%$ & $58.5 \%$ & $20.8 \%$ & $31.3 \%$ & $0.0 \%$ & $0.0 \%$ & $52.1 \%$ \\
\hline Summation & \multicolumn{2}{|c|}{$61.1 \%$} & \multicolumn{2}{|c|}{$38.9 \%$} & $100.0 \%$ & \multicolumn{2}{|c|}{$93.7 \%$} & \multicolumn{2}{|c|}{$6.3 \%$} & $100.0 \%$ \\
\hline
\end{tabular}

Percentage of patient observation point data (control group: 154, NSAID group: 48) in each group during observation period.

DAS 28-CRP : disease activity score28- c-reactive protein, NSAID : non-steroidal anti-inflammatory drug, RA : rheumatoid arthritis, MTX : methotrexate.

a Predonisolone equivalent. 
also increases MTX blood concentration. ${ }^{7)}$ The possibility of lowering MTX excretion in the kidney and development of serious adverse effects became highly probable in the patients of MTX treatment. ${ }^{5)}$ Therefore, we investigated the change of renal function such as BUN and eGFR in this study. The renal dysfunction was not observed; therefore the interaction mechanism between MTX and NSAIDs is considered to be their antiinflammatory interactions (pharmacodynamic interaction) rather than a pharmacokinetic interaction.

However, this study was a retrospective study; we have little data about the changes in the plasma MTX level and urine volume of patients investigated. The study on the effect of other DMARDs which prescribed before MTX treatment on MTX dosage, corticosteroid dosage, DAS 28-CRP and laboratory data would be also necessary.

When NSAIDs were previously prescribed to RA patients, the increase in MTX dosage could be avoided. It was suggested that NSAIDs treatment with MTX could control the RA activity without increasing MTX dosage, since it was reported that increased MTX dosage was related to adverse effects in RA patients. ${ }^{5)}$

In RA patients, the percentage of treatment combination with biological medicines such as etanercept and MTX for the induction of remission was gradually increased. ${ }^{18,19)}$ We will investigate the effect of etanercept on the dosage of MTX in the future. Recently, the MTX dosage was revised to increase the maximum dosage of MTX in RA from $8 \mathrm{mg} /$ week to $16 \mathrm{mg} /$ week in Japan. ${ }^{20)}$ Pharmacists should pay attention to the possibility of new drug interactions due to the increased MTX dosage.

\section{References}

1) Yamamoto K, Takasaki Y, Mimori T, Yamanaka H, "Medical manual of rheumatiod arthritis (revision); Diagnosis manual and treatment guideline based on EBM”, Ochi T, Japan Rheumatism Foundation, Tokyo, 2004, pp.63-104.

2) Pincus T, Yazici Y, Sokka T, Aletaha D, Smolen JS, Methotrexate as the "anchor drug" for the treatment of early rheumatoid arthritis, Clin Exp Rheumatol, 2003, 21 (Suppl. 31), S179-S185.

3) Shikata Y, Murai E, Ito M, Kitta T, Sakyo K, Hikida M, Shiba T, Kishi I, Evaluation of antirheumatic action of methotrexate (MTX) (1) Effects of MTX and 7-hydroxymethotrexate on collagen-induced arthritis and studies on the analgesic, anti-inflammatory and antipyretic action in rats, Pharmacomet, 1996, 52, 425-436.

4) Uwai Y, Suzuki R, Iwamoto K, Effect of nonsteroidal anti-inflammatory drugs on pharmacokinetics of methotrexate: A meta-analysis, YAKUGAKU ZASSHI, 2011, 131, 853-861.

5) Information of proper use of methotrexate (Rheuma$\operatorname{trex}^{\mathrm{TM}}$ ) $2 \mathrm{mg}$ in capsule, vol.16, Pfizer Corporation, Tokyo, 2011 Mar. revised edition, pp.10-14.

6) Nozaki Y, Kusuhara H, Kondo T, Iwaki M, Shiroyanagi Y, Nakayama H, Horita S, Nakazawa H, Okano T, Sugiyama Y, Species difference in the inhibitory effect of nonsteroidal anti-inflammatory drugs on the uptake of methotrexate by human kidney slices, J Pharmacol Exp Ther, 2007, 322, 11621170.

7) Sawada Y, Takanaga H, Yamada Y, Negishi E, Iga T, Drug interaction information 21, J Prac Pharm, 1999, 50, 1272-1288.

8) Bourre-Tessir J, Haraoui B, Methotrexate drug interactions in the treatment of rheumatoid arthritis: A systematic review, J Rheumatol, 2010, 37, 14161421.

9) Kremer JM, Hamilton RA, The effects of nonsteroidal anti-inflammatory drugs on methotrexate (MTX) pharmacokinetics: Impairment of renal clearance of MTX at weekly maintenance doses but not at 7.5 mg, J Rheumatol, 1995, 22, 2072-2077.

10) Prevoo MLL, van't Hof MA, Kuper HH, van Leeuwen MA, van de Putte LBA, van Riel PLCM, Modified disease activity scores that include twentyeight-joints counts: Development and validation in a prospective longitudinal study of patients with rheu- 
matoid arthritis, Arth Rheumatol, 1995, 38, 44-48.

11) American college of Rheumatology Subcomittee on Rheumatoid Arthritis Guidelines: Guidelines for the management of rheumatoid arthritis. Arth Rheumatol, 2002, 46, 328-346.

12) Kawasaki $Y$, Moriyama M, Shibata K, Gomita $Y$, The relationship between DMARD treatment and the dose of corticosteroids in rheumatoid arthritis therapy, J Jpn Soc Hosp Pharm, 2005, 41, 1539-1542.

13) Kawai VK, Grijalva CG, Arbogast PG, Curtis JR, Solomon DH, Delzell E, Chen L, Ouellet-Hellstrom R, Herrinton L, Liu L, Mitchel EF, JR, Stein MC, Griffin MR, Changes in cotherapies after initiation of disease-modifying antirheumatic drug therapy in patients with rheumatoid arthritis: Arth Care Res, 2011, 63, 1415-1424.

14) Yoshino S, Fujimori J, Koiwa M, Kishimoto M, A case of malignant rheumatoid arthritis with remarkable response to methotrexate, Ortho Surg, 1988, 14, 94-95.

15) Yonei $T$, Hagino H, Teshima R, Yamamoto K, Treatment of chronic rheumatoid arthritis with low-dose methotrexate, Ortho SurgTraum, 1991, 39, 1064 1067.

16) Ishioka $T$, Effect of loxoprofen sodium on renal function in erderly patients, Jpn J Clin Exp Med, 1990, 67, 272-274
17) Colebatch AN, Marks JL, Edwards CJ, Safety of non-steroidal anti-inflammatory drugs, including aspirin and paracetamol (acetaminophen) in people receiving methotrexate for inflammatory arthritis (rheumatoid arthritis, ankylosing spondylitis, psoriatic arthritis, other spondyloarthritis) (Review), Cochrane Lib, 2011, 11, 1-73.

18) van der Heijde D, Burmester G, Melo-Gomes J, Codreanu C, Mola EM, Pedersen R, Freundlich B, Chang DJ, The safety and efficacy of adding etanercept to methotrexate or methotrexate to etanercept in moderately active rheumatoid arthritis patients previously treated with monotherapy, Ann Rheumat Dis, 2008, 67, 182-188.

19) Emery P, Breedveld F, Hall S, Durez P, Chang DJ, Robertson D, Singh A, Pedersen RD, Koenig AS, Freundlich B, Comparision of methotrexate monotherapy with a combination of methotrexate and etanercept in active, early, moderate to severe rheumatoid arthritis (COMET): A randomized, doubleblind, parallel treatment trial, Lancet, 2008, 372, 375-382.

20) "Medical guideline of methotrexate (MTX) in rheumatoid arthritis treatment", Japan College of Rheumatology compiled, Yodosha, Tokyo, 2011, pp.19-27. 